\title{
Narrow-pass-band filters based on binary superlattices with strong impedance contrast
}

\author{
D.A. lakushev and N.M. Makarov \\ Benemérita Universidad Autónoma de Puebla, Puebla, Pue. 72000, México \\ A.Ya. Usikov Institute for Radiophysics and Electronics NASU, Kharkov 61085, Ukraine \\ E-mail: nykolay.makarov@correo.buap.mx \\ F. Pérez-Rodríguez \\ Benemérita Universidad Autónoma de Puebla, Puebla, Pue. 72000, México \\ E-mail: fperez@ifuap.buap.mx
}

Received January 27, 2017, published online June 26, 2017

\begin{abstract}
The photonic band structure and transmission coefficient of a dielectric bilayer periodic array with strong impedance contrast are calculated and analyzed. It is found that the photonic band structure has broad pass bands and very narrow gaps. However, because of the strong impedance contrast and the use of lossless dielectric layers, the transmission spectrum exhibits sharp peaks inside the pass bands. The remarkable properties of the superlattice can be used for the fabrication of narrow-pass-band filters.
\end{abstract}

PACS: 78.67.Pt $\quad$ Multilayers, superlattices, photonic structures, metamaterials;

42.70.Qs Photonic band gap materials;

78.67.-n Optical properties of low-dimensional, mesoscopic, and nanoscale materials and structures.

Keywords: multilayered metamaterials, strong impedance contrast, optical properties.

\section{Introduction}

Optical periodic nanostructures are of great importance for modern photonics because of the possibility to control the light propagation through them in a wide frequency range. Such nanostructures, being commonly composed of dielectric and metal components, have been called photonic metamaterials. They have promising applications in nanophotonics due to their exotic optical properties as, for example, hyperbolic photonic dispersion and negative refractive index [1-3].

In the case of one-dimensional periodic dielectric-metal nanostructures (i.e. superlattices), it is well established (see, e.g., [4-6]) that if the metallic layers are sufficiently thin then the photonic band structure exhibits narrow pass bands due to the very strong contrast between the impedances of the dielectric and the metal. The slightly dispersive (almost flat) photonic bands are associated with Fabry-Perot resonances occurring inside the relatively thick dielectric layer. However, the light transmission through a dielectric-metal multilayer stack diminishes with its length because of the energy losses in the metal component, which are particularly considerable in the $\mathrm{THz}$ and infrared frequency range.
Energy losses in optic superlattices with narrow photonic pass bands might be reduced by substituting the metal layers for dielectric ones of extremely high refractive index $[7,8]$. Indeed, the impedance of the ordinary dielectric layer, which has low enough refractive index, and that of the layer with high refractive index turn out to be essentially different and, therefore, sharp transmission resonances should be observed.

In this work the optical properties of one-dimensional periodic nanostructures composed of alternating dielectric layers with rather different impedances are investigated. In particular, the photonic band structure and transmission optical spectrum for binary dielectric superlattices are analyzed and discussed. We show that such periodic nanostructures with strong impedance contrast can operate as narrow-pass-band filters of electromagnetic radiation.

\section{Problem formulation: Basic relations}

We consider a plane electromagnetic wave propagating along the growth direction of a periodic multilayer stack, see Fig. 1. The wave has frequency $\omega$, its electric $\mathbf{E}(x, t)$ and magnetic $\mathbf{H}(x, t)$ components read

$$
\begin{aligned}
\mathbf{E}(x, t) & =\{0, E(x), 0\} \exp (-i \omega t), \\
\mathbf{H}(x, t) & =\{0,0, H(x)\} \exp (-i \omega t) .
\end{aligned}
$$




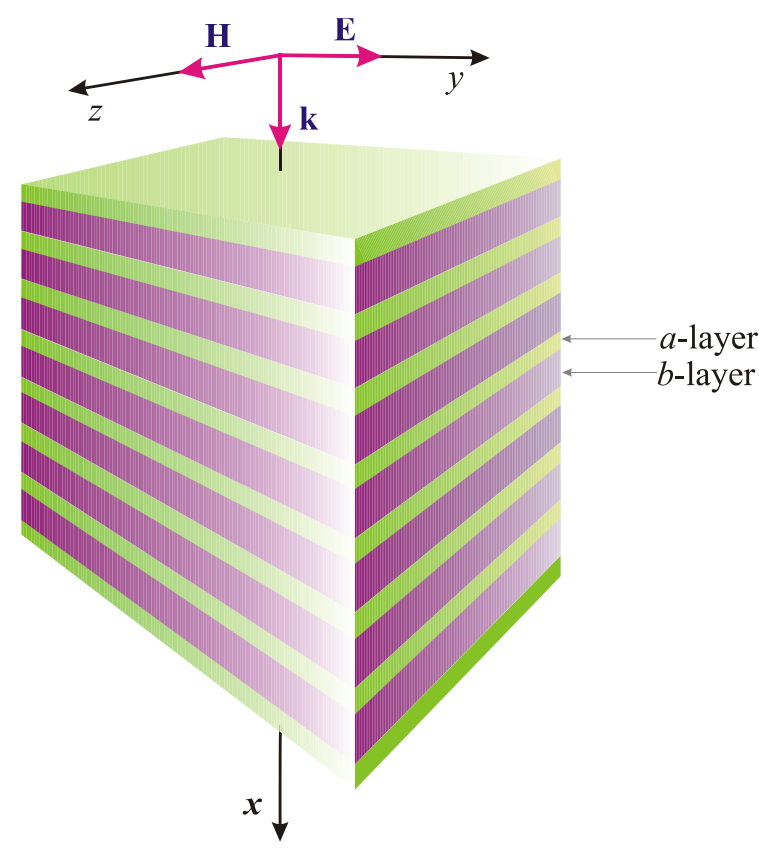

Fig. 1. (Color online) A sketch of the problem.

A unit $(a, b)$ cell of the stack consists of two dielectric $a$ and $b$-slabs with the constant thickness $d_{a}$ and $d_{b}$, respectively. The size of the unit cell is $d=d_{a}+d_{b}$. The array contains $N$ identical unit cells with the whole length $N d$.

Every kind of dielectric $a$ - and $b$-layers is respectively specified by the dielectric constant (permittivity) $\varepsilon_{a, b}$, magnetic permeability $\mu_{a, b}$, corresponding refractive index $n_{a, b}$, impedance $Z_{a, b}$, wave number $k_{a, b}$, and wave phase shift $\varphi_{a, b}$,

$$
\begin{aligned}
& n_{a}=\sqrt{\varepsilon_{a} \mu_{a}}, \quad Z_{a}=\frac{\mu_{a}}{n_{a}}, \quad k_{a}=\frac{\omega}{c} n_{a}, \quad \varphi_{a}=k_{a} d_{a} \\
& n_{b}=\sqrt{\varepsilon_{b} \mu_{b}}, \quad Z_{b}=\frac{\mu_{b}}{n_{b}}, \quad k_{b}=\frac{\omega}{c} n_{b}, \quad \varphi_{b}=k_{b} d_{b} .
\end{aligned}
$$

For definiteness, we assume that all optical parameters (2) are of positive values.

With the use of the transfer matrix method (see, e.g., the book [9]), the Bloch wave number $\kappa$ and the transmission coefficient (or, the same, the transmittance) $T_{N}$ of such a system can be readily obtained to obey two following equations:

$$
\begin{gathered}
\cos (\kappa d)=\cos \varphi_{a} \cos \varphi_{b}-\alpha_{+} \sin \varphi_{a} \sin \varphi_{b}, \\
T_{N}=\frac{1}{1+\alpha_{-}^{2} \sin ^{2} \varphi_{b} \sin ^{2}(N \kappa d) / \sin ^{2}(\kappa d)} .
\end{gathered}
$$

In these expressions

$$
\alpha_{ \pm}=\frac{1}{2}\left(\frac{Z_{a}}{Z_{b}} \pm \frac{Z_{b}}{Z_{a}}\right), \quad \alpha_{+}^{2}-\alpha_{-}^{2}=1,
$$

where the parameter $\alpha_{-}$is so-called mismatching factor. It is worth noting that the dispersion relation (3) defines the spectrum of the Bloch wave number, $\kappa=\kappa(\omega)$. Specifically, the Bloch wave number $\kappa$ turns out to be real inside the spectral pass bands (where $|\cos (\kappa d)| \leq 1$ ) and of complex value within the reflection bands (gaps with $|\cos (\kappa d)|>1$ ). In addition, one can reveals that expression (4) for the transmittance adequately describes the wave transmission both within spectral pass bands and gaps.

Equations (3) and (4) are quite general and applicable to the wide class of bilayer stack-structures. In the present study we shall analyze such systems whose unit-cell optical parameters are assumed to satisfy three constitutive conditions. First, the strong contrast between the impedances must be met: the impedance $Z_{b}$ of the $b$-layer must be much smaller than the impedance $Z_{a}$ of the $a$-layer,

$$
Z_{b}<<Z_{a} \rightarrow \alpha_{ \pm} \approx \frac{Z_{a}}{2 Z_{b}}>>1
$$

Second, the optic paths, $n_{a} d_{a}$ and $n_{b} d_{b}$, of the wave traveling through the corresponding basic $a$ - and $b$-slabs, are substantially different. However, as opposed to the condition (6), the optic path of the $b$-layer is assumed to be much greater than that of the $a$-layer, i.e.

$$
n_{a} d_{a}<<n_{b} d_{b} \rightarrow \varphi_{a}<<\varphi_{b}=\frac{n_{b} d_{b}}{n_{a} d_{a}} \varphi_{a} .
$$

Apart from the two parameters introduced above, great $Z_{a} / Z_{b}$, Eq. (6), and small $n_{a} d_{a} / n_{b} d_{b}$, Eq. (7), there is one more, a third parameter that is the product of the two previous ones. The third parameter has to be small: the first great ratio is compensated by the second small one,

$$
\frac{Z_{a}}{Z_{b}} \frac{n_{a} d_{a}}{n_{b} d_{b}}<<1 \rightarrow \frac{\mu_{a} d_{a}}{\mu_{b} d_{b}}<<1 .
$$

When the permeabilities $\mu_{a} \sim \mu_{b} \sim 1$, the third requirement (8) means that the thickness $d_{a}$ of the $a$-layer with smaller refractive index $n_{a}$ is much smaller than the thickness $d_{b}$ of the $b$-layer with the higher refractive index $n_{b}$. Therefore, three basic conditions (6)-(8) can be presented in the form of a double inequality,

$$
\frac{n_{a}}{n_{b}}<<1<<\frac{d_{b}}{d_{a}} .
$$

Note that this inequality is valid not only when $n_{a} \sim 1$ and $n_{b}>>1$, but also when $0<n_{a}<<1$ and $n_{b} \sim 1$.

\section{Spectrum}

The band structure of the spectral dependence $\kappa=\kappa(\omega)$ governed by the dispersion relation (3) starts with so-called lowest path band that is not of great interest. As for the higher bands, one can draw some important qualitative conclusions about them without actual solving the problem completely. Indeed, under the strong-contrast condition (6), the factor $\alpha_{+}$appearing in front of the product $\sin \varphi_{a} \sin \varphi_{b}$ 
in Eq. (3) has a great value. It is obvious that inside the pass bands, this great value should be compensated by very low values of $\sin \varphi_{a}$ or $\sin \varphi_{b}$. Hence, the transmission bands can be situated only in the vicinity of the $a$ - and $b$ resonances arising, respectively, in $a$ - and $b$-layers, i.e. in the vicinity of the points

$$
\varphi_{a}=j_{a} \pi \text { or } \varphi_{b}=j_{b} \pi,
$$

where $j_{a}$ and $j_{b}$ are positive integer numbers. Correspondingly, we shall call these bands $a$-band or $b$-band.

As a consequence of the condition (7), within the region between the top $\varphi_{b}=\pi$ of the lowest propagation band and the first $a$-resonance $\varphi_{a}=\pi$, i.e., when

$$
\pi \lesssim \varphi_{b} \lesssim \frac{n_{b} d_{b}}{n_{a} d_{a}} \pi,
$$

there are a lot of transmission $b$-bands associated with the $b$-resonances. At the same time, within the interval (11), the phase shift $\varphi_{a}$ changes from very small values, in the lower $b$-bands, to the values of the order of $\pi$, in the higher $b$-bands. Due to peculiar properties of the lower $b$-bands, which are demonstrated below, here we restrict ourself to the study of the lower $b$-bands only where the values of the phase shift $\varphi_{a}$ are small,

$$
\varphi_{a}=\frac{n_{a} d_{a}}{n_{b} d_{b}} \varphi_{b} \sim \frac{n_{a} d_{a}}{n_{b} d_{b}} j_{b} \pi<<1 .
$$

There exists common belief that the pass bands of a bilayer periodic structure with strong contrast of the impedances always should be narrow. Remarkably, the $b$-bands of the superlattice under consideration are expected to be broad, in spite of the strong contrast (6). Indeed, it easy to show that under conditions (6), (7) and within the frequency range (11), (12), the dispersion relation (3) can be represented in the following approximate form

$$
\begin{aligned}
\cos (\kappa d) & =\left[1-\frac{1}{2}\left(\frac{n_{a} d_{a}}{n_{b} d_{b}}\right)^{2} \varphi_{b}^{2}\right] \cos \varphi_{b}- \\
& -\frac{Z_{a}}{2 Z_{b}} \frac{n_{a} d_{a}}{n_{b} d_{b}} \varphi_{b} \sin \varphi_{b} .
\end{aligned}
$$

Surprisingly, the asymptotics (13) does not contain any great parameter, as opposed to initial Eq. (3). Moreover, the factor in front of $\varphi_{b} \sin \varphi_{b}$, turns out to be small due to condition (8). This is the reason why the $b$-bands are expected to be broad, whereas the gaps between them are expected to be narrow.

Within the zero approximation with respect to the second and third small parameters of the problem, see Eqs. (7) and (8), for the lower $b$-resonances (12), the simplified dispersion relation (13) degenerates into

$$
\cos (\kappa d)=\cos \varphi_{b} .
$$

In such a case the stack-structure is effectively equivalent to a homogeneous medium with linear spectrum and average refractive index $\bar{n}$. In the representation of extended Brillouin zones one gets

$$
\kappa d=\varphi_{b} \rightarrow \frac{\omega \bar{n}}{c}=\kappa, \quad \bar{n}=\frac{n_{b} d_{b}}{d} .
$$

Note that in this case there are no gaps in the spectrum. Due to the inequality (7), the average refractive index does not depend on the optic path $n_{a} d_{a}$ of $a$-layers.

In the first Brillouin zone representation, in which $|\kappa d| \leq \pi$, the spectrum has the form of a vertical "saw",

$$
\begin{gathered}
\frac{\omega \bar{n}}{c}=(-1)^{j_{b}} \kappa+\frac{2 \pi}{d}\left(\frac{j_{b}}{2}+\left\{\frac{j_{b}}{2}\right\}\right), \\
j_{b}=0,1,2, \ldots, \quad 0 \leq \kappa d \leq \pi,
\end{gathered}
$$

depicted in Fig. 2 by the dash-dot line. Here the braces $\{x\}$ refer to the fractional part of the inner number $x$. As $j_{b}$ is an integer, the fractional part $\left\{j_{b} / 2\right\}$ takes solely two values: it is zero for even $j_{b}$ and a half for odd $j_{b}$. Index $j_{b}$ enumerates the transmission $b$-bands: the larger $j_{b}$, the higher spectral $b$-band. The edges of the $b$-bands are defined by

$$
\begin{aligned}
& \varphi_{b}=0,2 \pi, 4 \pi, \ldots \quad \text { at } \quad \kappa d=0, \\
& \varphi_{b}=\pi, 3 \pi, 5 \pi, \ldots \quad \text { at } \quad \kappa d=\pi .
\end{aligned}
$$

As seen, the edges (17) of the unperturbed $b$-bands (16) are situated at the $b$-resonances (10). In the zero approximation, the width $\Delta \varphi_{b}^{\text {(band) }}$ of the $b$-bands is equal to $\pi$.

The zero-approximation band structure is degenerated because the top and the bottom of the neighboring unperturbed $b$-bands coincide. Accounting for the small terms

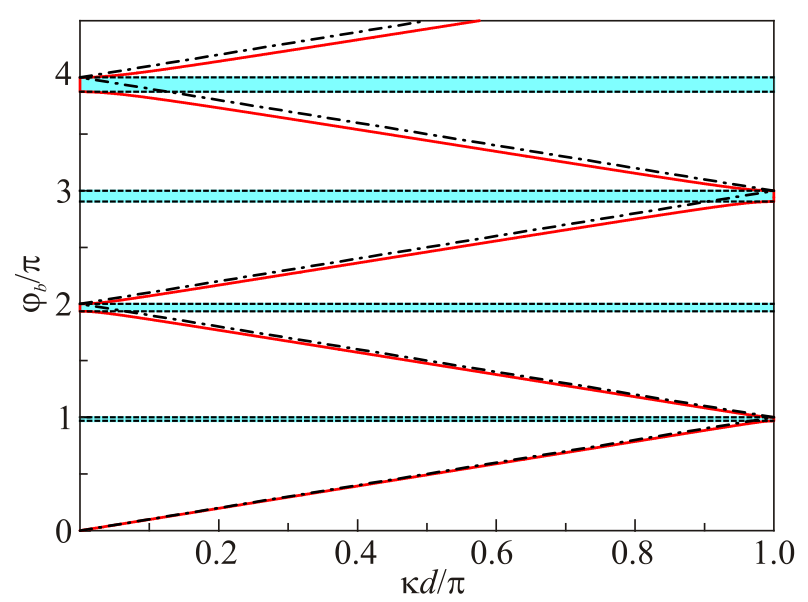

Fig. 2. (Color online) Solid line - band structure (3) for $d_{b} / d_{a}=30, n_{b} / n_{a}=25$. Dash-dot line - unperturbed band structure displayed by vertical "saw" (16). Horizontal dashed straight lines indicate the edges of the spectral broad bands and narrow gaps. 
in the dispersion relation (13) that have been omitted in Eq. (14), results in the non-zero width of the gaps as a consequence of the splitting of the $b$-band edges in the $b$-resonances.

For such a band structure, one might expect a practically perfect transmission for the whole spectrum except the narrow gaps. Remarkably, the analysis following below, completely disproves this supposition.

In the middle of the $b$-bands, the spectrum is linear. It can be approximately described by the vertical "saw" (16), see Fig. 2. Therefore, the analysis of the spectrum in the vicinity of the $b$-resonances, Eq. (17), is of actual interest only.

It can be shown that the width of the gaps is given by

$$
\Delta \varphi_{b}^{\text {(gap) }}=j_{b} \pi \frac{Z_{a}}{Z_{b}} \frac{n_{a} d_{a}}{n_{b} d_{b}}<<1 .
$$

The $b$-resonances are situated very close to the top edges of the gaps: the relative distance between the $b$-resonance and the top edge of the gap, with respect to the width of the gap is $\left(Z_{b} / Z_{a}\right)^{2}<<1$.

The width of the bands, $\Delta \varphi_{b}^{\text {(band) }}$, is found to be slightly smaller than $\pi$,

$$
\Delta \varphi_{b}^{(\text {band })}=\left[1-\left(j_{b}+1\right) \frac{Z_{a}}{Z_{b}} \frac{n_{a} d_{a}}{n_{b} d_{b}}\right] \pi .
$$

The bands are asymmetric because the corresponding $b$-resonances are placed within the bands, however, near their bottom edges: the ratio of the distance between the $b$-resonance and the bottom edge of the band to the width of the band is $j_{b} Z_{a} n_{a} d_{a} / Z_{b} n_{b} d_{b}<<1$.

To summarize, the stack-structure represents a quasihomogeneous medium. Specifically, the wide $b$-layers with high refractive index $n_{b}$ are separated from each other by the narrow $a$-layers with low refractive index $n_{a}$, i.e. $d_{a}<<d_{b}$ and $n_{a}<<n_{b}$, see Eq. (9). Therefore, within the zero approximation (neglecting $a$-layers), the spectrum of this structure is described by the linear dependence (15), or, the same, by the saw-like expression (16). The presence of the narrow $a$-layers gives rise to the emergence of the narrow gaps and slight changes of the spectrum at the edges of the broad bands whose width $\Delta \varphi_{b}^{\text {(band) }}$ is approximately equal to $\pi$. The exact band structure described by the exact dispersion relation (3) is illustratively compared with the unperturbed band structure (16) in Fig. 2.

\section{Transmittance}

With thorough analysis of the dispersion relation (3), we have shown that the band structure of the bilayer periodic array under consideration consists of broad bands separated by narrow gaps. At first glance, it seems that the system with such a band structure is perfectly transparent within the whole spectrum except the narrow gaps. Nevertheless, the analytical and numerical study of the expres- sion (4) for the transmittance, reveals that the behavior of the transmittance drastically differs from that based on the form of the band structure only. In fact, the transmittance has form of a "comb”: inside each pass band, the transmittance, getting very sharp peaks, turns out to be very small within large intervals between them. We show below that this unexpected effect is a direct consequence of the strong contrast condition (6). Within every $b$-band, the number of the peaks equals to the number $N$ of the unit $(a, b)$ cells that constitute the stack-structure. The leftmost peak is originated from the corresponding $b$-resonance (10). To the right from the $b$-peak, there are $(N-1)$ equidistant peaks contributed by the Fabry-Perot resonances,

$$
\kappa d=m \pi / N, \quad m=1,2,3, \ldots, N-1,
$$

arising due to multiple wave-reflections from the boundaries of the whole superlattice.

The predicted behavior of the transmittance can be understood as follows. By substitution of the unperturbed spectrum (15) into the general Eq. (4) for the transmittance $T_{N}$, one can readily express the latter in the terms of the phase shift $\varphi_{b}$,

$$
T_{N}=\frac{1}{1+\alpha_{-}^{2} \sin ^{2}\left(N \varphi_{b}\right)} .
$$

This equation shows that the transmission is perfect, i.e., $T_{N}=1$, when the phase shift $\varphi_{b}$ within the $j_{b}$ th $b$-band, takes values

$$
\begin{gathered}
\varphi_{b}^{(\text {res })}=\frac{m \pi}{N}, \quad m=1,2,3 \ldots \\
\frac{m}{N}=j_{b}, j_{b}+\frac{1}{N}, j_{b}+\frac{2}{N}, \ldots, j_{b}+\frac{N-1}{N} .
\end{gathered}
$$

Here the ratio $m / N=j_{b}$ defines the $b$-resonances, whereas the rest of the ratios, from $\left(j_{b}+1 / N\right)$ to $\left[j_{b}+(N-1) / N\right]$, refer to the Fabry-Perot resonances within the $j_{b}$ th $b$-band.

Between the peaks, the transmittance takes small values of the order of

$$
T_{N} \sim \alpha_{-}^{-2} \sim\left(\frac{Z_{b}}{Z_{a}}\right)^{2}<<1 .
$$

The small values of the transmittance between the peaks are caused by the mismatching factor $\alpha_{-}$which is great in the strong contrast regime, see Eq. (6). The value of $T_{N}$ given by Eq. (23) is inverse to the squared mismatching factor. Therefore, the transmittance $T_{N}$ decreases with increase of the mismatching factor rapidly enough.

Since the transmittance significantly differs from the small values of the order of $\alpha_{-}^{-2}<<1$ only in the narrow vicinities of the very sharp peaks situated at the resonances (22), we can determine the form of these peaks. By replacing the sine in Eq. (21) with its approximate expression in the vicinity of the resonances (22), we get 


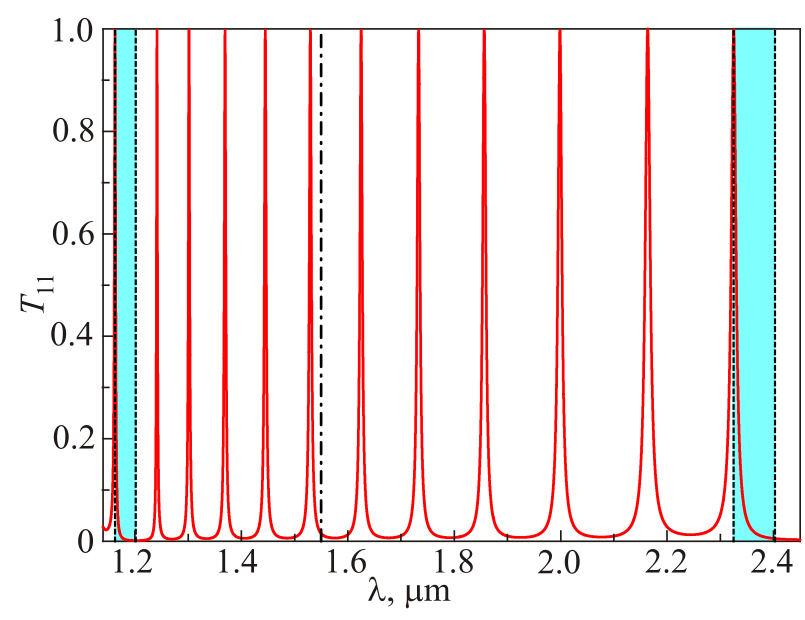

Fig. 3. (Color online) Transmittance (4) of the superlattice with number of unit cells $N=11$, within the second $b$-band (the band with $j_{b}=1$ that follows the lowest band with $j_{b}=0$ ) indicated in Fig. 2. Vertical dash-dot line shows wavelength $1.55 \mu \mathrm{m}$. Vertical dashed lines display the edges of the spectral gaps and bands.

$$
T_{N}=\frac{\Delta^{2}}{\Delta^{2}+\left(\varphi_{b}-\varphi_{b}^{(\mathrm{res})}\right)^{2}} .
$$

The peaks (24) have form of Lorentzian lines centered on $\varphi_{b}^{(\text {res) }}$ with the half width at half maximum

$$
\Delta=\frac{1}{N \alpha_{-}}<<1
$$

From Eq. (25) it follows that the sharpness of the peaks of the transmittance spectrum results from the inequality $N \alpha_{-}>>1$ which is always met in the strong contrast regime (6).

Figure 3 displays the resonant structure of the transmittance within the second $b$-band $\left(j_{b}=1\right)$ for the superlattice with $N=11$ unit $(a, b)$ cells, refractive indices $n_{a}=1$, $n_{b}=25$, and the relation between the layer thicknesses $d_{b} / d_{a}=30$. The thickness $d_{b}$ of the $b$-layer is chosen in such a way that the frequency corresponding to wavelength $1.55 \mu \mathrm{m}(1550 \mathrm{~nm})$, the standard wavelength for optical transfer-lines, is situated in the middle of the second band, where $\varphi_{b} / \pi=1.5$, see Fig 2 . This gives $d_{b}=46.5 \mathrm{~nm}$ and $d_{a}=1.55 \mathrm{~nm}$.

\section{Conclusions}

We have investigated the propagation of the electromagnetic radiation through the dielectric bilayer periodic array with strong contrast of impedances. Specifically, we have obtained and studied both the band structure and the transmission coefficient using analytical and numerical calculations.
We have found that, in spite of such a strong impedance contrast, lower $b$-bands are broad, whereas the gaps between them are narrow. This is because the strong contrast condition alone does not define the structure of the bands uniquely. Besides the strong contrast condition (6), there are two additional conditions (7) and (8) which reveal the structure of the pass bands. As for the transmission coefficient, we have clearly demonstrated that the array is not transparent within the almost whole spectrum. On the contrary, the transparency occurs in the quite narrow vicinity of the $b$ - and Fabry-Perot resonances only. In the intervals between these resonances, the transmission coefficient is very close to zero.

The remarkable properties of the superlattices described in the present study can be used for the fabrication of narrowpass-band filters. The main condition is using dielectrics with strong contrast of their impedances. For this purpose, as materials for the $a$ - and $b$-layers, one can respectively employ dielectrics with low and very high refractive index, $n_{a} \sim 1$ and $n_{b}>>1$. Another possibility is to use a low refractive index material with $n_{b} \sim 1$ for the $b$-layers and a material with unnaturally low refractive index, $0<n_{a}<<1$, for the $a$-layers.

\section{Acknowledgements}

This work was partially supported by the CONACYT (México) and by the PRODEP (México).

1. A. Poddubny, I. Iorsh, P. Belov, and Y. Kivshar, Nature Photon. 7, 948 (2013).

2. L. Ferrari, C. Wu, D. Lepage, X. Zhang, and Z. Liu, Progr. Quantum Electron. 40, 1 (2015).

3. C.M. Soukoulis and M. Wegener, Nature Photon. 5, 523 (2011).

4. A. Paredes-Juárez, D.A. Iakushev, B. Flores-Desirena, N.M. Makarov, and F. Pérez-Rodríguez, Opt. Exp. 22, 7581 (2014).

5. F. Diaz-Monge, A. Paredes-Juárez, D.A. Iakushev, N.M. Makarov, and F. Pérez-Rodríguez, Opt. Mater. Exp. 5, No. 2, 361 (2015).

6. A. Paredes-Juárez, D.A. Iakushev, B. Flores-Desirena, N.M. Makarov, and F. Pérez-Rodríguez, Opt. Lett. 40, 3588 (2015).

7. J. Arriaga, A.A Krokhin, and P. Halevi, Physica E 17, 436 (2003).

8. M. Choi, S.H. Lee, Y. Kim, S.B. Kang, J. Shin, M.H. Kwak, K.-Y. Kang, Y.-H. Lee, N. Park, and B. Min, Nature 470, 369 (2011).

9. P. Markoš and C.M. Soukoulis, Wave Propagation. From Electrons to Photonic Crystals and Left-Handed Materials, Princeton University Press, Princeton, NJ (2008). 